\title{
ARTÍCULOS
}

\section{Cicatrices de fuego en el leño de Prosopis caldenia en Luán Toro, provincia de La Pampa, Argentina}

\author{
Fire scars in Prosopis caldenia wood in Luán Toro, \\ La Pampa province, Argentina
}

\begin{abstract}
Andrea A Medina
Universidad Nacional de La Pampa, Facultad de Agronomía y Universidad Nacional del Comahue, Asentamiento Universitario, San Martín de Los Andes, Argentina, andrepampa@yahoo.com.ar
\end{abstract}

\begin{abstract}
SUMMARY
Fire is a significant component of the disturbance regime that affects Prosopis caldenia (caldén) woodlands with ecological and economical importance. Obtaining information on its temporal and spatial dynamics would be of great importance for management planning and rational use of these forests. The dating of fire scars registered in tree trunks is a widely used method for reconstructing fire regimes in forested areas. In this paper fire scars registered in the wood of caldén trees were analyzed from an area that was affected by prescribed fires to enhance pastures for livestock. Other marks, tree growth rings and structures associated to this disturbance are described. Although fire scars allow precise knowledge of the frequency and seasonality of past fires, the analysis of other structures associated to the influence of fire, such as ring separation, fire marks, "micro-rings" and rings with bands of parenchymatous tissue, would provide greater information on some immediate characteristics of fire regimes, such as intensity and spatial extent.
\end{abstract}

Key words: fire scars, fire marks, Prosopis caldenia.

\section{RESUMEN}

El fuego es un componente significativo del régimen de disturbios que afecta a los bosques de Prosopis caldenia (caldén), con importancia tanto ecológica como económica en los mismos. Contar con información sobre su dinámica, tanto temporal como espacial, resulta de gran importancia para la planificación del manejo y el uso racional del caldenal. El fechado de cicatrices de fuego registradas en los troncos es una metodología ampliamente utilizada para reconstruir regímenes de fuego en áreas boscosas. En este trabajo se analizaron las cicatrices de fuego registradas en el leño de caldenes de un bosque afectado por una serie de fuegos realizados con fines de manejo ganadero-pastoril y se describen otras marcas, anillos de crecimiento y estructuras asociadas a este disturbio. Si bien las cicatrices de fuego permiten conocer en forma precisa la frecuencia y la estacionalidad de los fuegos pasados, incluir el análisis de otras estructuras asociadas a la influencia del fuego, tales como separación de anillos, marcas de fuego, "microanillos" y anillos con bandas de tejido parenquimático, aportaría mayor información acerca de algunas de las características inmediatas del régimen de fuego, tales como su intensidad y extensión espacial.

Palabras clave: cicatrices de fuego, marcas de fuego, Prosopis caldenia.

\section{INTRODUCCIÓN}

El fuego es un componente significante del régimen de disturbios que afectan a los bosques de caldén (Prosopis caldenia Burk.), con importancia tanto ecológica como económica en los mismos. El bosque de caldén se encuentra en la provincia fitogeográfica del Espinal, la cual limita hacia el oeste con la provincia del Monte (Cabrera y Willink 1980). El bosque de caldén, el Monte y sus ecotonos sufren una alta ocurrencia de incendios en la región, tanto desencadenados por rayos de tormentas con- vectivas de verano, como de origen antrópico con fines de manejo ganadero-pastoril. La planificación del uso del fuego y su manejo racional en el caldenal necesita contar con información sobre la dinámica, tanto temporal como espacial, del fuego en este ecosistema boscoso.

El análisis dendrocronológico de cicatrices de fuego formadas en el leño de los árboles es una herramienta importante para reconstruir historias de fuego en los bosques, ya que éstas documentan tanto la fecha como la estación de ocurrencia del fuego, permitiendo así analizar la frecuencia, intensidad y extensión espacial de este 
fenómeno. Varias de estas características del régimen de fuego en los bosques de caldén han sido descritas a partir de la utilización de esta metodología (Medina et al. 2000, Medina 2003).

Las cicatrices de fuego se forman en los árboles cuando el fuego alcanza una temperatura letal para el cambium vascular (mayor a $60^{\circ} \mathrm{C}$ ), consumiendo en algunos casos la corteza, el cambium y porciones del xilema adyacente. Estas cicatrices son identificadas generalmente por la presencia de carbón y por bandas o rebordes de madera que cubren parcial o totalmente el área muerta (Mc Bride 1983). Estos rebordes corresponden al desarrollo de madera producida desde el sector del cambium vivo adyacente a la zona dañada y cuya consecuencia es la invasión y cobertura de este sector muerto por nueva madera. Varias especies del género Prosopis poseen la facultad de cubrir las heridas de fuego incrementando el crecimiento en los años subsiguientes desde la zona vecina a la herida (Bravo et al. 2001).

Las cicatrices de fuego pueden no presentar carbón en la herida cuando son originadas por fuego de baja intensidad (Guyette y Cutter 1997, Smith y Sutherland 1999, 2000).

En otros casos las lesiones producidas por fuego resultan más leves y el daño queda registrado a nivel de la corteza, manteniéndose la continuidad en la formación del tejido leñoso pero con cambios en su estructura (Giménez et al. 1997). Brown y Swetnam (1994) describen anillos y estructuras asociadas al fuego observadas en el leño de Sequoia sempervirens (Lamb.) Endl., como liberación del crecimiento, doble leño tardío, separación de anillos, presencia de conductos resiníferos y anillos de fuego o "microanillos".

Los árboles son marcados por numerosos agentes bióticos y abióticos. Por lo tanto, una reconstrucción precisa de la historia del fuego de un bosque requiere de la determinación correcta del fuego como la causa de las cicatrices de fuego y de anillos o estructuras asociadas al mismo.

El objetivo de este trabajo fue analizar las cicatrices de fuego (fecha y estación de ocurrencia) registradas en el leño de caldenes de un bosque afectado por una serie de fuegos realizados con fines de manejo ganadero-pastoril y describir otras marcas, anillos de crecimiento y estructuras asociadas a este disturbio.

\section{MÉTODOS}

Área de estudio. El área de muestreo es parte del predio Los Caldenes, cercano a la localidad de Luán Toro, en el departamento Loventué, en el norte de la provincia de La Pampa, Argentina.

Los bosques del área de estudio han sido históricamente afectados por fuego y desmonte con objetivos de explotación ganadera del sitio. Este tipo de explotación, actividad principal de la zona aún hoy, lleva al productor a quemar sus campos para inducir el rebrote tierno de pastos duros (Stipa tenuisima (Trinius), S. gynerioides (Philippi), para aumentar la cobertura de especies forrajeras (Stipa tenuis (Philippi), S. longiglumis (Philippi), Piptochaethium napostaense (Speg.) Hackel), Poa ligularis (Nees ex Steudel), para controlar la reaparición de leñosas y hasta en algunos casos para inducir el rebrote tierno de ramas de árboles y arbustos para consumo del ganado. Otro de los objetivos del uso de fuego en estos bosques es disminuir la masa combustible en los mismos para minimizar la probabilidad del paso de incendios destructivos por sus propiedades.

La precipitación en la zona es de régimen monzónico con una media anual de $500 \mathrm{~mm}$, produciéndose los mayores valores de déficit hídrico y de ocurrencia de tormentas convectivas en los meses de verano (diciembre a marzo). La temperatura media anual es de $15,5^{\circ} \mathrm{C}$, mientras que para los meses más fríos (junio a septiembre) es de $8^{\circ} \mathrm{C}$ y de $23,2^{\circ} \mathrm{C}$ para los meses de verano (Cano 1980).

Análisis de cicatrices de fuego. Se seleccionaron diez ejemplares de caldén con evidencias de múltiples cicatrices de fuego en sus troncos (figura 2A) en un área de 100 hectáreas de un bosque de caldén que ha sido afectado por una serie de fuegos realizados por el propietario del predio con fines de manejo ganadero-pastoril con intervalos de 4,2 años en promedio. La aplicación de fuego se realizó en febrero de 1971, enero de 1977, enero de 1980, diciembre de 1982, agosto de 1987 y octubre de 1992.

A los caldenes seleccionados se les extrajo una rodaja o sección transversal en la base de sus troncos. Estas rodajas fueron pulidas hasta lograr una clara visibilidad de los anillos de crecimiento. La observación y medición de estos anillos se realizó bajo lupa estereoscópica y considerando lecturas sobre dos a tres radios distintos. El año calendario de cada anillo de crecimiento corresponde al año en el cual el anillo comenzó a formarse (Schulman 1956).

Los anillos de crecimiento fueron cofechados mediante el uso del programa COFECHA (Holmes 1983) y se determinaron la fecha de ocurrencia y la estacionalidad de las cicatrices de fuego en base a su ubicación dentro del anillo: fuegos de primavera corresponden a cicatrices ubicadas en el leño temprano, fuegos de verano a las cicatrices ubicadas dentro del leño tardío y fuegos de otoño-invierno a las cicatrices ubicadas entre dos anillos de crecimiento consecutivos (Dieterich y Swetnam 1984, Baisan y Swetnam 1990).

Se describieron y fecharon otras marcas y anillos asociados a los eventos de fuego observadas en el leño de los caldenes analizados. 


\section{RESULTADOS}

Se observaron 24 cicatrices de fuego para el período 1971-1992, las que al ser fechadas reflejaron con exactitud las fechas (tanto el año como la estación) de las quemas realizadas e indicadas por el propietario (figura 1).

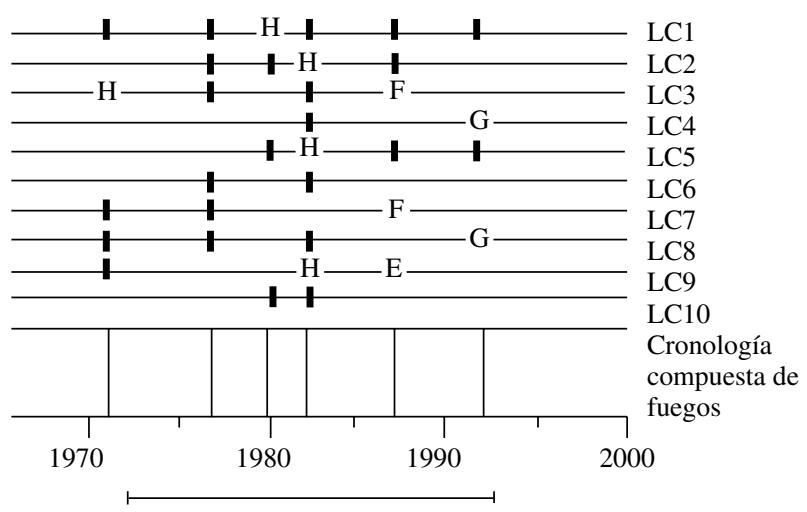

Período de análisis

Figura 1. Cronología de fuego del sitio y del período de estudio. Las líneas horizontales representan el tiempo de vida analizado de cada uno de los caldenes estudiados, en las cuales se representa el año en que fueron cortados los ejemplares (línea vertical corta a la derecha del gráfico), cicatrices de fuego (líneas verticales gruesas), anillos con bandas de tejido parenquimático $(\mathrm{H})$, separación de anillos (E), marcas de fuego (F) y "microanillos" (G). Debajo, cronología compuesta de fuego del período analizado, en la cual las líneas verticales indican la ocurrencia de cicatrices de fuego en al menos un ejemplar analizado en el área de estudio.

Fire chronology of the study period and site. Horizontal lines correspond to the life time analyzed for each sample until death year (indicated by short vertical bars at the end of the series), fire scars (dark vertical bars) and other fire-associated wood features or ring series $(\mathrm{H}, \mathrm{E}$, F y G). Below, the composite fire chronology of analyzed period, where vertical bars indicate fire occurrence in at least one sample.

La totalidad de las cicatrices de fuego presentó marcas de carbonización. Los fuegos ocurridos en los meses de diciembre, enero y febrero quedaron registrados con cicatrices en medio del leño tardío (figura 2B), mientras que las cicatrices originadas por el fuego del mes de octubre de 1992 se ubicaron en el leño temprano del anillo correspondiente (figura 2C), pudiéndose observar los vasos del leño temprano con rasgos de carbonización. El fuego ocurrido en el mes de agosto de 1987 produjo cicatrices de fuego en el límite entre los anillos de crecimiento de los años 1986 y 1987 (figura 2D).

En el material estudiado se observó la presencia de anillos y estructuras asociadas a la influencia del fuego (figuras 1 y 2), tales como separación de anillos (figura $2 \mathrm{E}$ ), marcas de fuego (figura $2 \mathrm{~F}$ ), microanillos (figura 2G) y anillos con bandas de tejido parenquimático en su interior (figura $2 \mathrm{H}$ ).

\section{DISCUSIÓN Y CONCLUSIONES}

El fechado de las cicatrices de fuego del período analizado refleja con exactitud las fechas (tanto el año como la estación) de las quemas realizadas e indicadas por el propietario del establecimiento. Este resultado demuestra la eficacia del análisis de cicatrices de fuego para desarrollar reconstrucciones de este disturbio en los bosques de caldén.

En fuegos de baja intensidad, las cicatrices de fuego pueden no estar asociadas con la presencia de carbón en la herida (Guyette y Cutter 1997, Smith y Sutherland 1999, 2000). En este estudio la totalidad de las cicatrices de fuego $(\mathrm{N}=24)$ presenta marcas de carbonización, lo cual podría estar asociado a la intensidad de las quemas. Por razones de manejo (principalmente el control de leñosas), los propietarios realizan quemas de moderada a alta intensidad, mayormente en meses de primavera tardía y verano. Este es el momento en que los árboles están en pleno crecimiento y desarrollo del leño, encontrándose más susceptibles a la incidencia del fuego (Llorens 2000).

Los fuegos ocurridos en los meses de diciembre, enero y febrero quedaron registrados en cinco de los diez caldenes analizados por anillos de crecimiento con bandas de tejido parenquimático en su leño tardío (figura $2 \mathrm{H})$. Esta proliferación de tejido parenquimático estaría asociada a la formación de un tejido de cicatrización como reacción al daño producido en la zona cambial. Brown y Swetnam (1994) describen una estructura asociada al fuego que denominan "doble leño tardío", la que estaría representando cambios ecofisiológicos desencadenados por fuego, similares a los procesos que resultan en una liberación del crecimiento. Las respuestas del crecimiento a modificaciones ambientales durante la formación de leño tardío pueden conducir a una breve producción de células de las del tipo del leño temprano antes de la formación final anual de leño tardío. Este tipo de estructura no pudo ser observada en el leño de los caldenes analizados en el presente trabajo.

El fuego ocurrido el mes de agosto de 1987 produjo cicatrices de fuego en el límite entre los anillos de crecimiento de los años 1986 y 1987 (figura 2G) en dos de los 10 árboles analizados. Esto se debe a que la formación del leño tardío del año 1996 ya había concluido (la caída de las hojas del caldén se completa entre fines de abril y principios de mayo) y aún no había comenzado a formarse el leño temprano del año 1987 (el caldén brota entre la última quincena de octubre y los primeros quince días de noviembre) (Cano 1980). En uno de los árboles analizados este fuego produjo una separación entre los anillos de crecimiento de los años 1986 y 1987 (figura 2E). La separación de anillos puede corresponder a un área donde el cambium ha sido afectado por calentamiento, aunque no se ha desarrollado una cicatriz característica (Brown y 

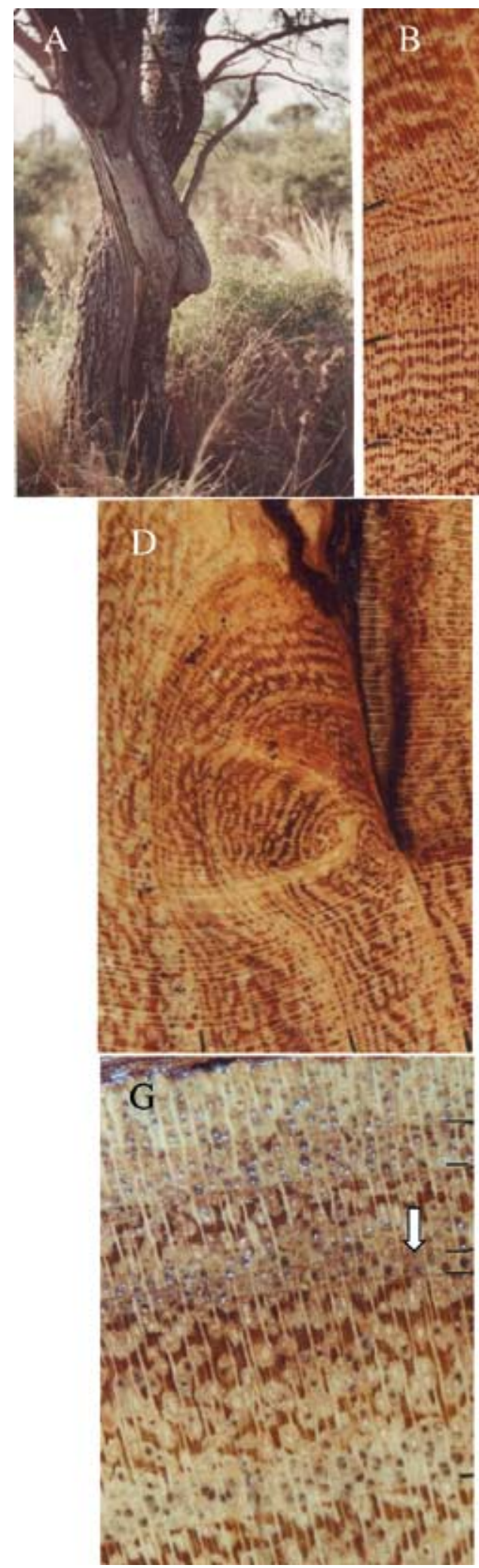
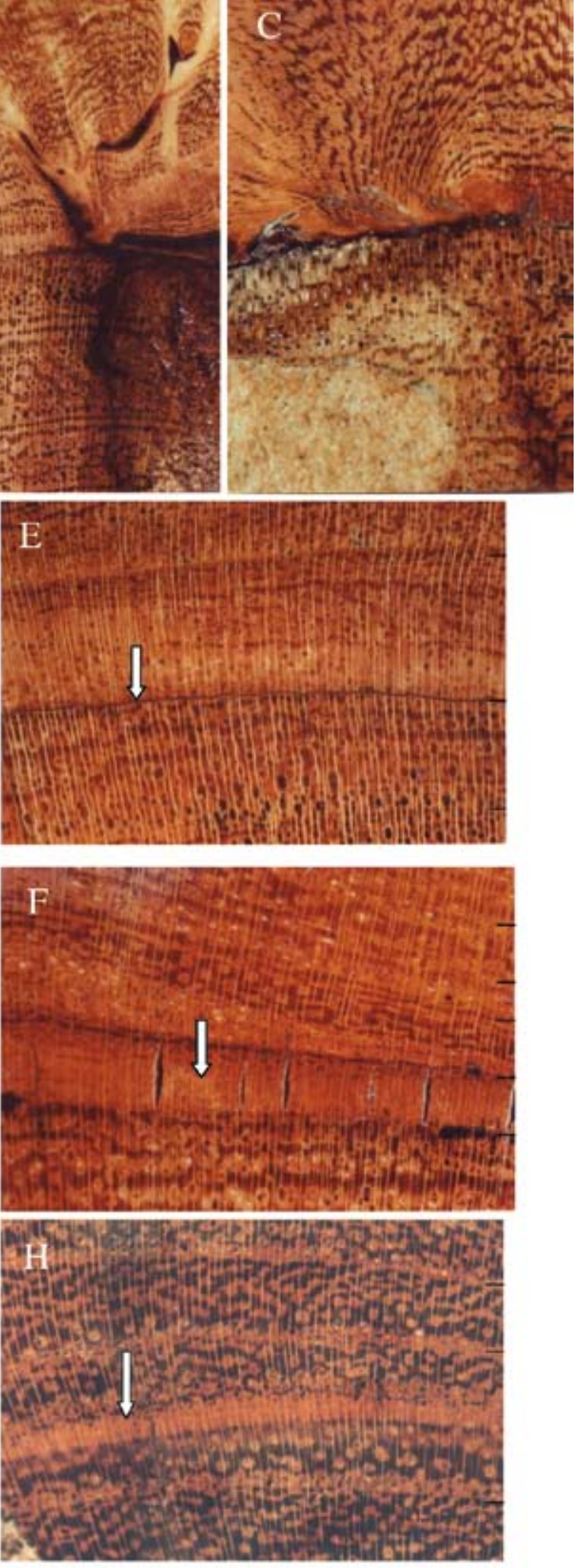

Figura 2. A) Caldén con múltiples cicatrices de fuego; B) cicatriz originada por el fuego realizado en febrero de 1971; C) en octubre de 1992; D) en agosto de 1987; E) separación de anillos de crecimiento originada por el fuego de agosto de 1987; F) marca de fuego originada por el fuego de agosto de 1987; G) "microanillo" del año 1992; H) anillo de crecimiento del año 1979 con abundante tejido parenquimático dentro del sector de leño tardío.

A) Calden with multiple fire scars; B) fire scars originated by the February 1971 fire; C) by the October 1972 fire; D) by the August 1987 fire; E) growth ring separation originated by the August 1987 fire; F) fire mark originated by the August 1987 fire; G) "micro-ring" of year 1992; H) growth ring with abundant parenchymatic tissues in its latewood, originated by the January 1980 fire. 
Swetnam 1994). Otra estructura observada asociada a este fuego invernal es la que Bravo et al. (2001) denominan como "marcas de fuego". Estas son descritas como lesiones más leves que las cicatrices de fuego características, identificándose macroscópicamente por un oscurecimiento en uno o más sectores del anillo de crecimiento, sin carbonización del tejido cambial ni leñoso y con continuidad en la formación de tejido leñoso pero con cambios en su estructura (figura $2 \mathrm{~F}$ ).

El fuego del mes de octubre de 1992 quedó registrado en dos de los diez caldenes con la formación de un anillo poco desarrollado (figura $2 \mathrm{G}$ ). Esta estructura fue denominada por Brown y Swetnam (1994) como fire rings (anillos de fuego) y podría estar vinculada a incidencias en la producción de la fotosíntesis en detrimento del cambium vascular.

Si bien las cicatrices de fuego, identificadas en general por la presencia de carbón y de bandas o rebordes de madera que cubren parcial o totalmente el área cambial muerta (Mc Bride 1983), son las evidencias más utilizadas en las reconstrucciones de historias de fuego, la inclusión de otras marcas o estructuras y anillos asociados a este disturbio puede aportar mayor información acerca de algunas de las características inmediatas del régimen de fuego, tales como su intensidad y extensión espacial.

\section{AGRADECIMIENTOS}

A Alejandro Gorondi, por su apoyo logístico y aporte de información.

\section{REFERENCIAS}

Baisan CH, TW Swetnam. 1990. Fire history on a desert mountain range: Rincon Mountain Wilderness, Arizona, U.S.A. Canadian Journal of Forest Research 20: 1559-1569.

Bravo S, AM Giménez, JG Moglia. 2001. Efectos del fuego en la madera de Prosopis alba Griseb. y Prosopis nigra (Griseb.) Hieron, Mimosaceae. Bosque 22(1): 51-63.

Brown PT, TW Swetnam. 1994. A cross-dated fire history from coast Redwood National Park, California. Canadian Journal of Forest Research 24: 21-31.
Cabrera AL y A Willink. 1980. Biogeografía de América Latina. Serie de Biología, Monografía $\mathrm{N}^{\circ} 13$. Washington DC, EE.UU. Secretaría General de la Organización de los Estados Americanos, Programa Regional de Desarrollo Científico y Tecnológico. 117 pp.

Cano E. 1980. Inventario Integrado de los Recursos Naturales de la provincia de La Pampa. Clima, Geomorfología, Suelo y Vegetación. Buenos Aires, Argentina. Universidad Nacional de La Pampa, Instituto Nacional de Tecnología Agropecuaria. 493 p.

Dieterich JH, TW Swetnam. 1984. Dendrochronology of a firescarred Ponderosa Pine. Forest Science 30: 238-247.

Giménez AM, N Ríos, G Moglia. 1997. Leño y corteza de Itin Prosopis kuntzei (Harms) en relación a algunas magnitudes dendrométricas. Investigaciones Agropecuarias: Sistemas y Recursos Forestales 6(1 y 2): 163-182.

Guyette RP, BE Cutter. 1997. Fire history, population, and calcium cycling in the current river watershed. In Pallardy SG, RA Cecich, HG Garrett, PS Johnson, eds. Proceedings of the $11^{\text {th }}$ Central Hardwood Forest Conference. Gen. Tech. Rep. NC-188. St. Paul, MN. US Department of Agriculture, Forest Service, North Central Forest Experiment Station. p. 354-372.

Holmes RL. 1983. Computer-assisted quality control in tree-ring dating and measurement. Tree-Ring Bulletin 43: 69-75.

Llorens EM. 2000. Estrategias de manejo del Caldenal para lograr una máxima producción sustentable. Nuestro Campo 13-17.

Mc Bride JR. 1983. Analysis of tree rings and fire scars to establish fire history. Tree-Ring Bulletin 43: 51-67.

Medina AA, EG Dussart, HD Estelrich, EA Morici. 2000. Reconstrucción de la frecuencia de fuego en un bosque de Prosopis caldenia Burkart, de Arizona, sur de la Provincia de San Luis. Multequina 9: 91-98.

Medina A. 2003. Reconstrucción de historias de fuego en bosques mediante técnicas dendrocronológicas. In Kunst CR, S Bravo, JL Panigatti eds. Fuego en los Ecosistemas Argentinos. Santiago del Estero, Argentina. Instituto Nacional de Tecnología Agropecuaria. p. 133-143.

Schulman E. 1956. Dendroclimatic change in semiarid America. Tucson, Arizona. University of Arizona Press. 142 p.

Smith KT, E Sutherland. 1999. Fire-scar formation and compartmentalization in oak. Canadian Journal of Forest Research 29: 166-171.

Smith KT, E Sutherland. 2000. Oak biology and fire scar formation. Abstracts of the International Conference on Dendrochronology for the Third Millennium, Laboratorio de Dendrocronología, Mendoza, Argentina. p. 39. 